\title{
High-Resolution Cryo-SEM of Latex Film Formation
}

\author{
Haiyan Ge*, H. Ted Davis*, L. E. Scriven* \\ University of Minnesota, Chemical Engineering \& Material Science Dept., Minneapolis MN 55455
}

As a latex coating — a deposited layer of colloidal particles of polymer suspended in solvent—dries, it is transformed into a coherent solid coating. This happens in three stages, which may overlap: (1) solvent evaporation and particle packing, or consolidation; (2) particle deformation and pore-space shrinkage, or compaction; and (3) particle adhesion and inter-particle diffusion of polymer, or coalescence. The details of this transformation process cannot be visualized by light microscopy when, as is usually true, the latex particles are a few tens or hundreds of nanometers in size, nor by conventional electron microscopy, which requires high vacuum that is unattainable until all of the solvent has evaporated.

Pioneering studies of Sutanto and Ma [1,2] showed that time-sectioning high-resolution cryogenic scanning electron microscopy is an unparalleled technique of visualizing this transformation process in detail. This technique consists of rapidly freezing samples of coatings after successively longer periods of drying, fracturing them to expose cross-sections, subliming small amounts of frozen liquid from the fracture surface to produce topographical contrast, applying the usual thin metal coating, and imaging them by a field emission SEM (FESEM) equipped with cold stage. With the Hitachi S4700 available to us, the resolution of latex film formation has been refined to about $5 \mathrm{~nm}$.

Except at the layer's edges, the several stages of transformation from colloidal suspension to coalesced coating usually proceed from the top down, i.e., from the exposed surface of the coating to the buried interface with its substrate, or support. Thus the process inherently involves gradients perpendicular to the substrate. At the edges, the process tends to proceed from the very edge inward (somewhere merging with the top-down succession); the process near the edges inherently involves gradients nearly parallel to the substrate. These gradients warrant investigation.

Time-sectioning cryo-FESEM was used to study the top-down and edge-in gradients of consolidation, compaction, and coalescence stages in model systems of monodisperse acrylate latex particles around 110 nanometers in diameter; glass transition temperature of the polymer in bulk is around room temperature $\left(20^{\circ} \mathrm{C}\right)$. The images, as exemplified by the coating dried 21 minutes before freezing (see Figure 1), show that consolidation is followed by compaction and compaction by coalescence- both from top toward bottom and from edge toward center. Whether all three stages coexist depends on size and viscoelastic properties - hence glass transition temperature — of the polymer particles, on the thickness of the coating, and on the drying conditions, viz. temperature, humidity, and velocity of air. In one case, after only 17 minutes, the coating is merely consolidated: spherical particles are in contact with ice that filled the porespace between them. After 30 minutes, the coating is compacted: particles have flattened against each other and make a hexagonal pattern in the fracture surface, little porespace remains, and the fracture has plastically drawn stalks out of individual particles. After 60 minutes, individual particles are rarely identifiable and the fracture draws out longer scattered ridges, which proves to be characteristic of coalescing coating. The rich three-dimensionality of the fracture surface is revealed by stereo images in Figure 2. 


\section{References}

[1] E. Sutanto, Liquid Distribution in Porous Media, PhD thesis, (1993) University of Minnesota.

[2] E. Sutanto et al., ACS Symposium Series (2001), 790 (Film Formation in Coatings), 174.

[3] The specially selected latex from U. Dittmer of BASF and the aid of S. L. Erlandsen and C. D. Frethem of University of Minnesota are gratefully acknowledged.

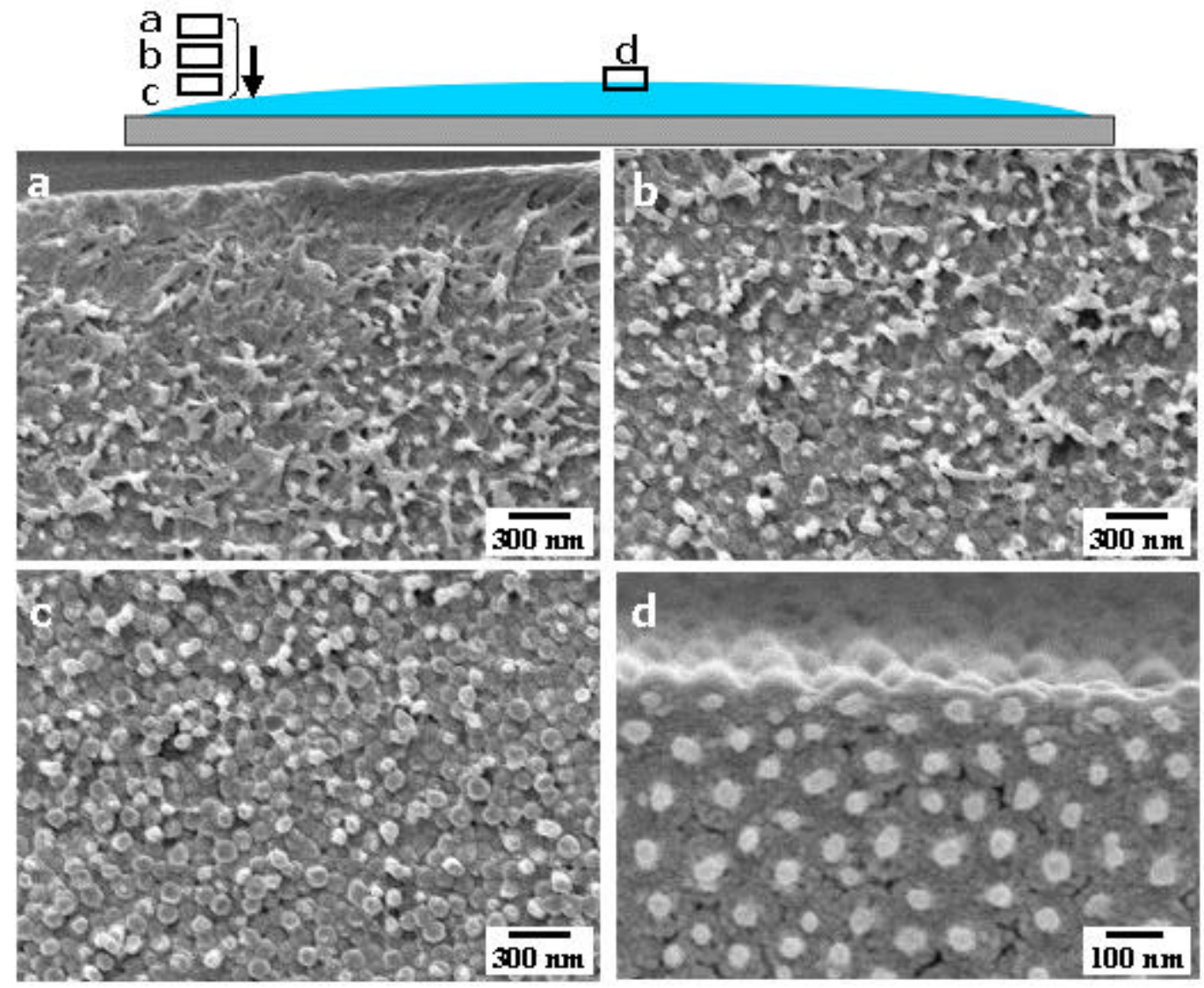

Figure1 In the thinner layer near the edge of a coating dried 21 minutes at room temperature all three stages are present in (a), (b), (c), whereas in the thicker middle (d) even the upper region is still consolidating.

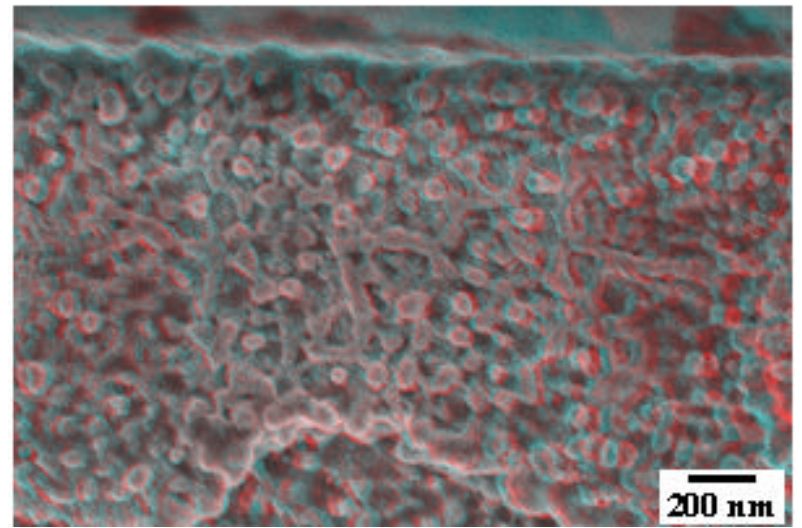

Figure 2 Stereo images of another sample coating dried 60 minutes at room temperature, showing vagaries of the fracture surface and the characteristic plastically drawn ridges. 